\title{
Conflictos bioéticos en investigación clínica: una perspectiva histórica
}

Agustín Estévez

El Código de Nuremberg cerraba un pasado vergonzoso para la investigación clínica. $\mathrm{E}$ iniciaba el intento de reivindicar el valor ético y social de la experimentación como un momento esencial del progreso médico. Se trataba de mostrar que para conseguir una buena ciencia era indispensable establecer ciertos procedimientos que encuadraran la experimentación sobre humanos en los límites de la tradición de la ética médica. La medicina nazi era una pesadilla que el llamado Juicio a los doctores cerraba para siempre y que limpiaba en adelante el camino a los buenos médicos, bastaba la honestidad profesional para garantizar una buena práctica de investigación y para asegurar a la humanidad sufriente los mejores resultados. Sin embargo en los hechos las cosas no resultaron tan sencillas. Por de pronto no había pautas ni criterios para juzgar las aberraciones cometidas por los científicos nazis. Su defensa en los 
Juicios a los doctores, fue que no habían violado ninguna ley, ya que ni en Alemania ni en el extranjero había normas que regularan sobre la investigación en humanos. Y en muchos sitios se hacían investigaciones violando vulnerabilidad y consentimiento de los humanos. ¿Cómo juzgar entonces aquellas investigaciones cuyos diseños aún teniendo valor científico sometían a prisioneros de guerra o a grupos humanos a mutilaciones o directamente a la muerte? ${ }^{1}$ Se tuvo que acudir para sancionar estos excesos al recurso del derecho natural y a combinar tradiciones humanísticas angloamericanas y alemanas, para disponer de unos principios que pudieran legitimar las sanciones. Había que admitir principios universales para juzgar estos excesos. Esto tenía consecuencias que marcarían con fuerza la evolución posterior de la historia de la investigación clínica. Se admitía que hay bienes humanos que tienen que ser protegidos más allá de los límites de las legislaciones positivas. Hay exigencias fundamentales que son derechos humanos imprescriptibles y que configuran derechos morales que tienen que pensarse como transculturales.

Los diez principios que forman el cuerpo del Código de Nuremberg $(\mathrm{CN})$ son mucho más que pautas orientativas, constituyen principios sustanciales con pretensión de universalidad. Esto es particularmente cierto del consentimiento voluntario que el $\mathrm{CN}$ lo considera "absolutamente esencial". Como se verá más tarde de él depende la investigación con sujetos humanos, y desde aquí se instaura un nuevo paradigma para la relación entre paciente-sujeto y médico-investigador. Es esta pretensión de transculturalidad que modifica la significación histórica del CN. Ya no se trata de cerrar una historia de horrores arreglando cuentas con un pasado que no tiene que volver a repetirse. Ni de

\footnotetext{
Véase para la descripción de estos experimentos y las cuestiones bioéticas que plantean: Caplan, A. (ed.). When Medicine IVent Mad, Totowa, New Jersey, Humana Press, 1992.
} 
concebir sus principios como una legislación ad hoc para juzgar crímenes que no estaban contemplados por las legislaciones nacionales. Se sabe de los esfuerzos de los doctores Andreuw Ivy y Leo Alexander ${ }^{2}$ para llenar las lagunas que existían en la época de los Juicios, todavía no había un código unívoco de principios de ética de investigación. Al resolver cuestiones puntuales con un pragmatismo de urgencia, se pusieron, como a veces suele suceder, exigencias de validez que le proporcionaron al $\mathrm{CN}$ una significación atemporal. En adelante la validez de los principios trascendía el momento histórico de su establecimiento para devenir exigencias permanentes. Los principios se constituían en un canon con el cual se podría en adelante medir las desviaciones que se cometiesen en investigación clínica. Todo esto lo testimonia en primer lugar la reticencia con que los profesionales de la salud alemanes tomaron los resultados del juicio. Muchos médicos que habían tenido graves responsabilidades no sufrieron ningún castigo y siguieron cumpliendo funciones, inclusive públicas en el gobierno de la Alemania Federal. ${ }^{3}$

En el momento de la celebración de los Juicios los gremios médicos enviaron un representante para que testimoniara sobre las acusaciones. El Dr. Alexander Mitscherlich que desempeñó este cometido, se sorprendió de cómo grandes figuras de la medicina habían cometido actos de crueldad inaudita. Sus testimonios jamás fueron comentados, inclusive la publicación de sus notas desaparecieron, y recién en la década del sesenta apareció su libro. ${ }^{4}$

\footnotetext{
${ }^{2}$ Leo Alexander y Andreuw Ivy fueron los dos consultores médicos en los Juicios a los médicos. El último que representaba a la Asociación Médica Americana desarrolló una intensa tarea para encontrar un marco universal para encuadrar los juicios. ${ }^{3}$ htpp: $/ /$ www.apiavirtual.com/modules.php?name $=$ News\&file $=$ article\&sid $=4007$.

${ }^{4}$ Alexander Mitscherlich// Fred Melke (eds.). Medizin ohne Menschlichkeit. Dokumente des Nümberger Árzteprozesses, Frankfurt, 1960.
} 
La exigencia de universalidad de los principios del $\mathrm{CN}$ la muestra por contraste lo que se llama la "brecha postNuremberg", los propios países que impusieron el CN violaron en muchas de sus investigaciones los principios que habían establecido. Era como si ese requisito de universalidad y autonomía del $\mathrm{CN}$ costaba adquirirlo, y retornaba el viejo paternalismo médico. Los médicos y científicos norteamericanos que no eran nazis, pensaban que el $\mathrm{CN}$ no se aplicaba a ellos, que sólo quedaba restringido a los nazis. Ellos eran honestos y virtuosos por eso obrarían de acuerdo a cánones éticos y científicos para bien de la humanidad. Como se sabe los hechos desmintieron estas aseveraciones, poco a poco los profesionales se fueron percatando que los principios de CN le concernían y que la autonomía era el principio clave que protegía al sujeto pero también hacía posible la empresa de la investigación misma.

\section{Universalismo y autonomía}

¿En qué consiste la universalidad de los principios de Nuremberg? Uno de los problemas del mundo contemporáneo reside en lo difícil que resulta articular en una visión moral común la diversidad de formas de vida buena que proponen diferentes culturas. El relativismo reinante mas la persistencia de un pensamiento que se esfuerza en seguir fragmentando el mundo hace para muchos dudosa la tarea. Sin embargo todavía tiene vigencia bajo el amparo de los derechos humanos una tradición ilustrada que constituye el aspecto más esperanzador y útil para la legitimación de la ética aplicada. Nos referimos contemporáneamente a distintas posturas de pensamiento, como las éticas del discurso, los neocontractualismos, y diferentes intentos de fundamentación y justificación de normas, que parten de concebir 
la moral como la resolución pacífica de conflictos. ${ }^{5}$ Tal perspectiva supone condiciones de simetría y reciprocidad entre los agentes, que permiten concebir un entramado de vínculos recíprocos que hacen posible un factor común, por lo menos de conexión débil.

Los autores que establecieron el $\mathrm{CN}$ se inspiraron en tradiciones liberales, iusnaturalistas y contractualistas, es por esto que resulta casi natural interpretar las exigencias que establece Nuremberg con el principio de autonomía que constituye el núcleo fundamental de esta tradición.

En el CN el primer artículo establece sin ambigüedad el primado indiscutible del consentimiento informado como procedimiento de autorización para participar en un proyecto de investigación. No caben dudas que esta formulación se hace a la luz de los brutales experimentos nazis que forzaban a las personas, exponiéndolas a situaciones de mutilaciones, dolor y muerte. Pero el estilo en que está redactado "...es absolutamente esencial" revela claramente que la intención es la de establecer un principio sustancial. Una norma ética fuerte que modificará en adelante la relación entre investigador y sujeto.

Dos cuestiones queremos destacar. En primer lugar el consentimiento es el procedimiento que realiza el principio de autonomía. Por lo tanto tiene una significación ético-jurídica. Es un proceso de comunicación y asentimiento que culmina con la autorización para una intervención sobre el propio cuerpo. Pero en segundo lugar, la autonomía es el fundamento que autoriza, pudiendo ser interpretada como una elección no coaccionada entre opciones sobre las que se le informa al paciente o sujeto de investigación. Su significado reside en la capacidad de un sujeto para tomar

\footnotetext{
${ }^{3}$ Nos referimos a autores como Appel, Habermas y sobre todo Rawls. En la tradición moderna Locke, Kant, Rousseau. Pero también a autores más difíciles de filiar como Tugenhat.
} 
por sí mismo aquellas decisiones que lo afectan en su salud e integridad, generalmente se exige también que ha de conformarse al estilo de vida del sujeto. En bioética prima esta interpretación, que llamaríamos política de la autonomía, en la que se considera que lo que se trata de obtener son acciones autónomas, o decisiones que las reflejen. No es éste el sentido del concepto de autonomía, cuando es referido al fundamento de la moral o al origen de la obligación. La autonomía en esta significación remite a un querer originario de la conciencia moral (Gewissen) que configura la decisión originaria y autoconciente por la moral, decisión absolutamente libre porque siempre esta la posibilidad de optar contra la moral. Esta decisión básica esta por debajo de toda moral concreta y es tan antigua como la Regla de Oro. En efecto, se es autónomo cuando se decide por la moral y ésta aparece como una red de reciprocidad entre conciencias. Esta reciprocidad es ambigua porque puede concebírsela como intercambio interesado y condicionado, o ser pensado desinteresadamente sin buscar compensaciones ni devoluciones, adoptando una concepción independiente de lo bueno. Este sentido de reciprocidad, moral y razonable, constituye lo universal de los principios mismos. Formalmente un principio moral es universal porque no hace acepción de personas, vale para todos los individuos de la misma clase por igual.

El sentido estricto de autonomía concibe al orden moral, no como algo dado, sino como algo puesto por una decisión originaria por la moral. Cualquier fundamento heterónomo que se sugiriese, tiene que evaluarlo la propia conciencia moral (decidida). Surge aquí una necesidad hipotética de la autonomía, donde se apoya en última instancia la autorización con la que culmina el consentimiento informado.

Robert Baker en un interesante artículo, ha intentado fundamentar la universalidad de los principios del CN, 
a partir de una interpretación peculiar de la teoría del contrato. Por un lado reconoce que lo valores posiblemente compartibles de la teoría contractualista: “...igualdad de los contrayentes, rol legitimante del asentimiento, y en última instancia el valor universal de los derechos humanos" ${ }^{\prime \prime}$, no parecen ser tan decisivos como la conciencia del conflicto que subyace a los acuerdos. El carácter universal y transcultural de los principios de Nuremberg, presupone conflictos compartidos y su exitosa resolución. En efecto, el consentimiento informado resolvía la ambigüedad fundamental en la relación entre médico-investigador, paciente-sujeto de investigación, y validaba por la autorización del interesado experimentos sobre sujetos sanos. No es en cambio lo que sucedió con la ética de la virtud y la Declaración de Helsinki de 1964 que retornaron a un indefendible paternalismo médico que culminó con investigaciones no éticas, mostrando el fracaso de la autorregulación por parte de médicos e investigadores. De todas maneras nos parece que esta interesante visión de destacar el momento del conflicto no contradice, la de concebir la autonomía en su sentido fuerte. En todo caso le agrega un criterio de aplicación y validez a los principios que podrían ser compartidos.

\section{Consecuencias en la investigación clínica del consenti- miento informado}

En la ética médica tradicional nunca se legitimó la investigación que no tuviera intención terapéutica. ${ }^{7}$ Por eso la experimentación era casual y episódica. No se concebía que se expusiese a un ser humano sano a los rigores de un protocolo. 8

\footnotetext{
${ }^{6}$ Robert Baker (1997), pág.22

'Remitimos al interesante capitulo de Diego Gracias Investigación clínica. Estudios de Bioética, 4, (1998).

${ }^{8}$ Piénsese la fase I para probar la toxicidad de un nuevo fármaco.
} 
El principio de beneficencia y de no maleficencia regían la relación médica, el bien del paciente era la meta objetiva y social de la práctica terapéutica. Por supueșto que ese beneficio era mirado exclusivamente desde la perspectiva profesional del médico. Regía en la ética médica el paternalismo beneficentista y en el ámbito del conocimiento el saber clínico. Todavía no se había tomado conciencia de la importancia de la autonomía de los pacientes.

Fue el progreso de la tecnología médica lo que exigió cambios; por un lado el reconocimiento de los derechos de los pacientes, los que como personas y agentes morales debían ser protegidos en su integridad, en su privacidad y en sus estilos de vida y decisiones personales. Por el otro la insuficiencia del puro saber clínico, más intuitivo que verificado por los cánones científicos e intersubjetivos. Esto se evidenciaba particularmente en el campo de los medicamentos. Casos trágicos y paradigmáticos mostraban la necesidad de la investigación clínica previa a la comercialización de un nuevo fármaco. ${ }^{9}$ En adelante la evidencia clínica requería de la validación científica y de la investigación clínica generalizada. La llamada medicina de la evidencia extendía estas exigencias a todo el campo de atención de la salud.

¿Pero cómo legitimar éticamente el empleo generalizado de sujetos humanos en investigación? Un solo principio y un único procedimiento justificaba el uso instrumenta I izado del ser humano: su consentimiento, su autonomía. Únicamente una decisión informada hacía moralmente posible y justificable la experimentación clínica. Desde Nuremberg en adelante el consentimiento deviene una condición esencial e imprescindible en una buena práctica de investigación. Constituyendo la condición de posibilidad ética de la investigación clínica.

\footnotetext{
${ }^{9}$ Como lo mostró el famoso caso de la talidomida.
} 


\section{Avances y Retrocesos}

El CN estableció pautas éticas sustanciales, sus principios constituyen un marco insuperable para la dimensión ética de la investigación clínica. En particular con el Consentimiento Informado se abría un nuevo dominio en la historia de la investigación biomédica.

En un sentido más inmediato su intención residía en restablecer la dignidad de la ciencia y la investigación clínica frente a los excesos de los doctores nazis. Era posible realizar una buena ciencia si se respetaban los principios clásicos de la ética médica. Había que terminar con la pesadilla de la medicina nazi. El CN parecía ante todo referirse exclusivamente a aquellas prácticas bárbaras que nada tenían que ver con los buenos médicos que se formaban en el rigor de sus disciplinas. El paternalismo clásico de la profesión médica se legitimaba con una pretendida ética de la virtud que no precisaba de molestas regulaciones externas, proviniesen éstas de la sociedad o de los legos y pacientes. ${ }^{10}$

Los médicos norteamericanos e ingleses consideraron que no se requería para el ámbito terapéutico el Consentimiento Informado. Su honestidad y la buena práctica médica lo suplían. Por eso fue muy bien recibida la distinción que estableció la Declaración de Helsinki de 1964 entre investigación terapéutica y no terapéutica. Los principios éticos fueron concebidos aquí como pautas orientativas de uso opcional para médicos y científicos. El Consentimiento Informado quedaba como requisito para la experimentación no terapéutica. La preferencia y adopción de una ética de la virtud hacía innecesaria toda regulación externa que

\footnotetext{
${ }^{10}$ Baker destaca la renuencia de los médicos de Harvard a aceptar el consentimiento informado indicado por el $\mathrm{CN}$, y la satisfacción de científicos y profesionales con la distinción entre investigación terapéutica y no terapéutica instituida por la Declaración de Helsinki de 1964. En el mismo sentido destaca Diego Gracia, el retorno al patemalismo médico que subyace a esa distinción.
} 
se consideraba una intromisión dentro de la relación médica. Se acudía a la propia responsabilidad de los profesionales, a la conciencia de su excelencia.

Esta postura hegemónica fue quebrada por una serie de graves abusos en investigaciones en Estados Unidos. Se mostró que en cuestiones como la salud, y la experimentación humana se requerían regulaciones externas, control de agencias estatales, y participación ciudadana. Es así que se establecieron una serie de Comisiones Presidenciales en las que se trato cuestiones que requerían un tratamiento público y participativo. Resultado de una Comisión fue el Informe Belmont, que al establecer por primera vez los principios que orientasen la práctica ética de la investigación clínica, constituían la carta de nacimiento de la bioética. Este documento rescataba el sentido ético sustancial de Nuremberg y lo extendería a toda la práctica médica. Los principios que en el Informe estaban limitados a la investigación se generalizarían luego a la práctica médico-hospitalaria. Nacía así la bioética médica que se constituía en una visión crítica del poder de las profesiones de la salud. El principio de autonomía se transformaba en una poderosa arma de la crítica contra el paternalismo médico, para preservar al paciente como persona y sujeto moral se requería ahora del auxilio de agencias estatales y de nuevas instituciones de ciudadanía que como los comités de ética proponían modelos interdisciplinarios y con participación ciudadana para dar respuestas a los desafíos que ponían a la ética el progreso y dominio de la tecnología médica.

En todo caso lo que se había visto es que la investigación no podía quedar en manos exclusivas de científicos y médicos, porque los resultados se parecían demasiado a lo que Nuremberg condenaba. Esto por supuesto constituía un desafío para los legos y ciudadanos, una obligación de participar, de ilustrarse para tener respuestas que no sólo tuvieran una excelencia profesional, sino también calidad ética. 
La historia de la investigación clínica reciente nos muestra que continúa la contraposición entre posturas que garantizan la protección del paciente-sujeto y aquellas que pretenden regulaciones más flexibles para incrementar la libertad de acción de investigadores y promotores. Se trata de una historia abierta en la que la bioética intenta colocar un espacio de diálogo para que todas las partes expongan sus razones y a veces puedan proponerse regulaciones más razonables y éticas.

\section{BIBLIOGRAFÍA}

Baker, R.: "Un modelo teórico para la ética médica transcultural: postmodernismo, relativismo y el Código de Nuremberg" en Perspectivas Bioéticas en las Américas, Año 2, N mero 1, Primer Semestre de 1997, FLACSO, Argentina.

Beauchamp,T. and Childress, J.: Principles of Biomedical Ethics, New York, Oxford University Press, 1994.

Caplan, A. (ed.). When Medicine Went Mad, Totowa, New Jersey, Humana Press, 1992.

EstÈvez, AgustÌn: Bioética, de la cuestión nominal a la caracterización de su concepto, Bahía Blanca, Editorial de la Universidad Nacional del Sur, 2002.

Gracia Guillen, Diego: "Investigación Clínica" en Profesión Médica, investigación y Justicia sanitaria, Estudios de Bioética, volumen 4, Bogotá, El Buho, 1998.

Joñas, Hans: Técnica, Medicina y ética, Barcelona, Paidós, 1997.

Kant, I.: Fundamentación de la Metafísica de las Costumbres, ed. bilingüe de J. Mardomingo, Barcelona, Ariel, 1999.

Rawls, J.: Liberalismo político, México, Fondo de Cultura, 1995.

Tealdi, J. C.: "Documentos de Deontología Médica" en Mainetti, José A.: Etica Médica, Introducción Histórica, La Plata, Quirón, 1989.

Tugenhat, E.: Lecciones de ética, Barcelona, Gedisa, 1997.

Tugenhat, E.: "Cómo debemos entender la moral?", en Problemas, Barcelona, Gedisa, 2002 